Document downloaded from:

http://hdl.handle.net/10251/101124

This paper must be cited as:



The final publication is available at

https://doi.org/10.1016/j.cemconcomp.2017.02.009

Copyright Elsevier

Additional Information 


\title{
Ultrasonic broadband signals monitoring of glass-fiber reinforced cement (GRC) bending tests
}

\author{
V. Genovés ${ }^{\mathrm{a}, *}$, J. Gosálbez ${ }^{\mathrm{b}, * *}$, A. Carrión ${ }^{\mathrm{b}}$, R. Miralles ${ }^{\mathrm{b}}$, J. Payá $^{\mathrm{a}}$ \\ ${ }^{a}$ ICITECH, Universitat Politècnica de València, Camino de Vera, s/n 46022 Valencia, Spain \\ ${ }^{b}$ ITEAM, Universitat Politècnica de València, Camino de Vera, s/n 46022 Valencia, Spain
}

\begin{abstract}
In this study, complete ultrasonic monitoring of Glass-fiber Reinforced Cement plates under bending tests was addressed. In this kind of experiment, the mechanical properties of the specimen continuously change during the test, thus, the acquisition time of the ultrasonic signals is a critical variable. In order to overcome this drawback, a new ultrasonic procedure based on broadband signals (chirp) has been applied. Following this line of thought, the analysed ultrasonic parameters have been split into the parameters that only depend on time, and those that depend on both time and frequency. In particular, the frequency dependent attenuation parameter allows characterizing the evolution of the plate being damaged over a wide frequency range and significantly detecting the main two events happening during the experiment: the first crack and the maximum stress point. In short, this paper demonstrates the suitability of ultrasonic broadband signals for characterizing fiber-reinforced cementitious composites under bending stress.
\end{abstract}

Keywords:

Process monitoring, Reinforced cement, Ultrasonics, Mechanical testing, Glass fibres

\section{Introduction}

Glass-fiber reinforced cement (GRC) is a composite made of Portland cement mortar with a low water/cement $(\mathrm{w} / \mathrm{c})$ ratio with a high percentage of paste (water + cement) in relation to the aggregate. The addition of a high proportion of alkali resistant (AR) glass fibers to the mortar matrix (3\%-5\% by weight of mortar) improves the mechanical properties of the composite, particularly its toughness, flexural strength, and ductility. This composite has an important role in non-steel reinforced pre-cast concrete elements, such as sheets, panels and other slim shapes usually employed in building engineering and architecture, as well as in cast-in-situ sprayed-on surfaces $[1,2]$.

Recently, non-destructive techniques (NDT) applied to concrete have been investigated, especially ultrasonics and acoustic spectroscopy, in order to obtain parameters related to the physical and mechanical properties of

\footnotetext{
*Corresponding author E-mail address: genoves.gomez@gmail.com

**Corresponding author E-mail address: jorgocas@dcom.upv.es
} 
the material and its durability [3]. Some experimental studies have demonstrated that wave parameters such as the ultrasonic pulse velocity of P-waves are suitable for predicting the dynamic elastic modulus of the concrete, and that the velocity of the S-waves can predict the dynamic shear modulus. These parameters are proportional to the elastic and shear moduli of concrete and also to its compressive strength $[4,5]$. Other studies based on ultrasound propagation indicate that wave attenuation should be measured for various frequencies, in order to characterise cement-based materials, determining their microstructure, porosity, and other characteristics in both the hardened [6-9] and fresh states [10].

Some studies of construction material tests by ultrasound monitoring have been reported in the literature. Many of them are related to research on metallic specimens because this type of material is used in other engineering fields. However, recent studies of bending tests of reinforced concrete slabs, using ultrasonic monitoring, have been carried out successfully $[11,12]$ and other kind of important processes like concrete setting $[13,14]$. The first approach was recently published by the authors, in which the monitoring of GRC plates under a four point bending test using an ultrasonic pulse with a fixed frequency was carried out. Thus, the P-wave velocity, attenuation, energy and non-linear parameters were obtained [15]. Monitoring mechanical tests with nondestructive techniques provides a wealth of information about the material under study, allowing the prediction of its behaviour. Ultrasonic waves could be appropriate for following the changes during the mechanical test. GRC plates change their internal structure because micro-cracks develop during the bending test. In these conditions, different ultrasonic frequencies are needed to cover all the changes in the specimen. The disadvantage of this kind of test is the fact that the mechanical properties of the GRC plates change quickly during the test, and it is difficult to inject various signals with different frequencies to cover a wide spectrum. To solve this problem, the authors have also recently published a study of the suitability of broadband signals, comparing different methods and signals to obtain a reliable method to acquire the information of a wide bandwidth with one single signal (chirp) [16]. This procedure permits monitoring a huge range of situations where the test-time has critical effects. The aim of this paper is to apply new ultrasonic acquisition techniques to support the previous results and provide new information about the GRC plates under stress, making use of the attenuation and non-linear ultrasonic parameters in the time and frequency domain.

The remainder of this paper is structured as follows. Section 2 describes the materials and specimens used in this study. It also presents the test layout, composed of the ultrasonic equipment and the mechanical test machine, as well as the ultrasonic parameters extracted from the signals during the experiments. In Section 3, the results are presented, correlating the ultrasonic parameters with mechanical curves. Finally, in Section 4, the conclusions are summarized. 


\section{Experimental}

\subsection{Materials and specimens}

In this experiment, the specimen dimensions for the bending test were $325 \times 50 \times 20 \mathrm{~mm}$. The specimens were cut from a $400 \times 400 \times 20 \mathrm{~mm}$ mother plate, obtaining five plates for the tests. Table 1 shows the proportions and information about the raw materials used to fabricate the GRC mother plate. The specimens were made according to the BS EN 1170-5 standard [17].

Table 1: Used dosage for one GRC plate specimen

\begin{tabular}{lll}
\hline Material & Type & Weight $[\mathrm{g}]$ \\
\hline Cement & CEM I 52.5 R & 7260 \\
Water & - & 2541 \\
Sand & Silica sand 0/2 & 4864 \\
Superplasticizer & Polycarboxylate ether & 14 \\
Fiber & Glass AR 12 mm length & 294 \\
\hline
\end{tabular}

\subsection{Experimental layout}

An ultrasonic through-transmission setup was selected due to its penetration capability and accuracy for velocity and attenuation estimation [6, 18-20]. The transducers used for transmission and reception were K1SC (General Electric). Both are broadband transducers with a bandwidth centered at $1 \mathrm{MHz}$. Universal testing machine (Instron model 3382) was used for the four point bending test. The distance between the supports was $295 \mathrm{~mm}$ for the passive supports and $98 \mathrm{~mm}$ for the active ones. The displacement of the actuator was constant during the test $(0.6 \mathrm{~mm} / \mathrm{min})$. The transmitter transducer was excited directly by a programmable signal generator (Agilent 33120A).

In order to do a broadband analysis, the transmitted signal was a swept-frequency signal (chirp). The use of chirp signals enables estimating any parameter in a wide frequency range with a single measurement by applying the proper signal processing. The mathematical expression of the transmitted linear chirp signal is

$$
\begin{gathered}
s_{t x}(t)=A_{t x} \cos \left(2 \pi f_{0} t+\pi \Delta_{f_{\max }} t^{2}\right) \operatorname{rec} t\left(\frac{t-\frac{T_{w}}{2}}{T_{w}}\right) \\
\Delta_{f_{\max }}=\frac{f_{\max }-f_{0}}{T_{w}}
\end{gathered}
$$

where $A_{t x}$ is the amplitude of the signal, $f_{0}$ is the fundamental frequency, $\operatorname{rect}(\cdot)$ is the rectangular function, $T_{w}$ is the active time of the signal, and $\Delta_{f_{\max }}$ controls the maximum frequency $\left(f_{\max }=f_{0}+T_{w} \Delta_{f_{\max }}\right)$ which is reached at $T_{w}$ seconds (Eq. 2). The selected parameters for the transmitted chirp signal are $A_{t x}=10 \mathrm{~V}, T_{w}=10 \mu \mathrm{s}$ and the frequency range varies from 200 to $1000 \mathrm{kHz}$. The experiment was monitored by injecting chirp signals with a period $(T)$ equal to 0.5 seconds. Fig. 1 shows a time line of the test, where three signals are represented: a 


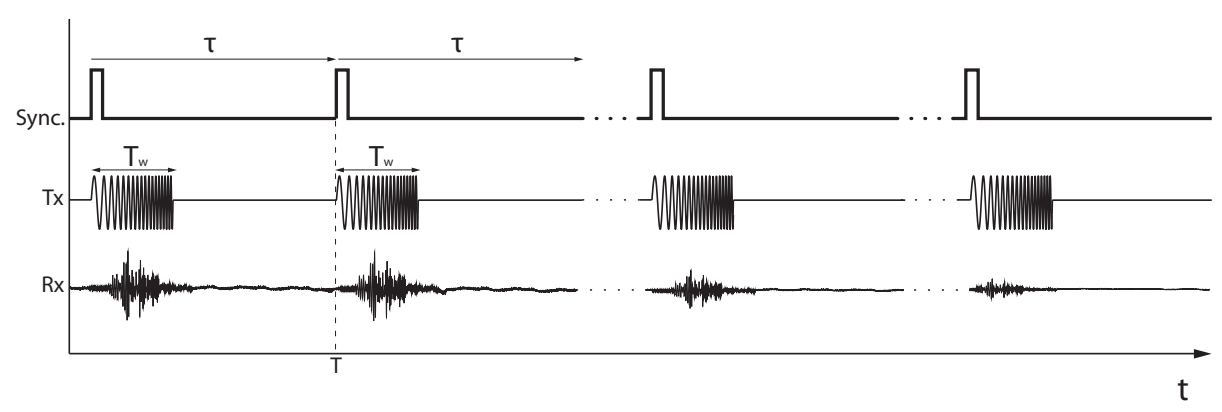

Figure 1: Time line of the test where three signals are represented: A synchronization signal between events (Sync.), and the transmitted (Tx) and received (Rx) signals, as well as some of the variables used in the mathematical analysis ( $\tau, T$ and $T_{w}$ ).

signal of synchronization between events (Sync.), the transmitted (Tx) and received (Rx) signals, as well as some of the variables used in the mathematical analysis.

The reception transducer was connected to a linear $40 \mathrm{~dB}$ preamplifier (Panametrics 5600B). The received and amplified ultrasonic signal was captured by a digital oscilloscope (Tektronix DPO3014) with a sampling frequency of $25 \mathrm{MHz}$. Finally, a laptop was used to control the signal generator and to store the digitized signals.

The experimental layout of the mechanical test is shown in Fig. 2. The plate rests on the passive supports and four metallic angles were attached to the plate in the positions shown. These elements serve to hold the elastic bands which keep constant the pressure between the faces of the transducers and the specimen. A similar system was presented in [21], yielding consistent results and suitable performance, where the rubber bands kept the appropriate pressure between the transducers and specimen. Pure vaseline was used to ensure an appropriate coupling between the transducers and the faces of the specimen.

\subsection{Ultrasonic parameters}

Using broadband signals in the analysis allows computing some ultrasound parameters which not only vary in the time domain (during the test) but also in the frequency domain. In order to support the previous results, only the time dependent parameters are also analysed. In the following, the analysed ultrasonic parameters are classified into two groups: parameters dependent on the time alone, and parameters dependent on both time and frequency.

\subsubsection{Time dependent parameters}

Many parameters were extracted from the received signals, giving information about the material for every instant of time. Let $x_{t, r x}$ be the amplitude of the received signal at time $t$, and let $\tau$ be the time variable in the interval from 0 to $T$ seconds (Fig. 1). The propagation velocity, $v_{p}(t)$, is obtained as the ratio between the length of the specimen, $d_{\text {mat }}$, and the time of arrival of the signal, $\tau_{t, a}$ (Eq. (3)), which was estimated as the point in time when $x_{t, r x}$ exceeds $50 \%$ of the noise level.

$$
v_{p}(t)[m / s]=\frac{d_{m a t}}{\tau_{t, a}}
$$




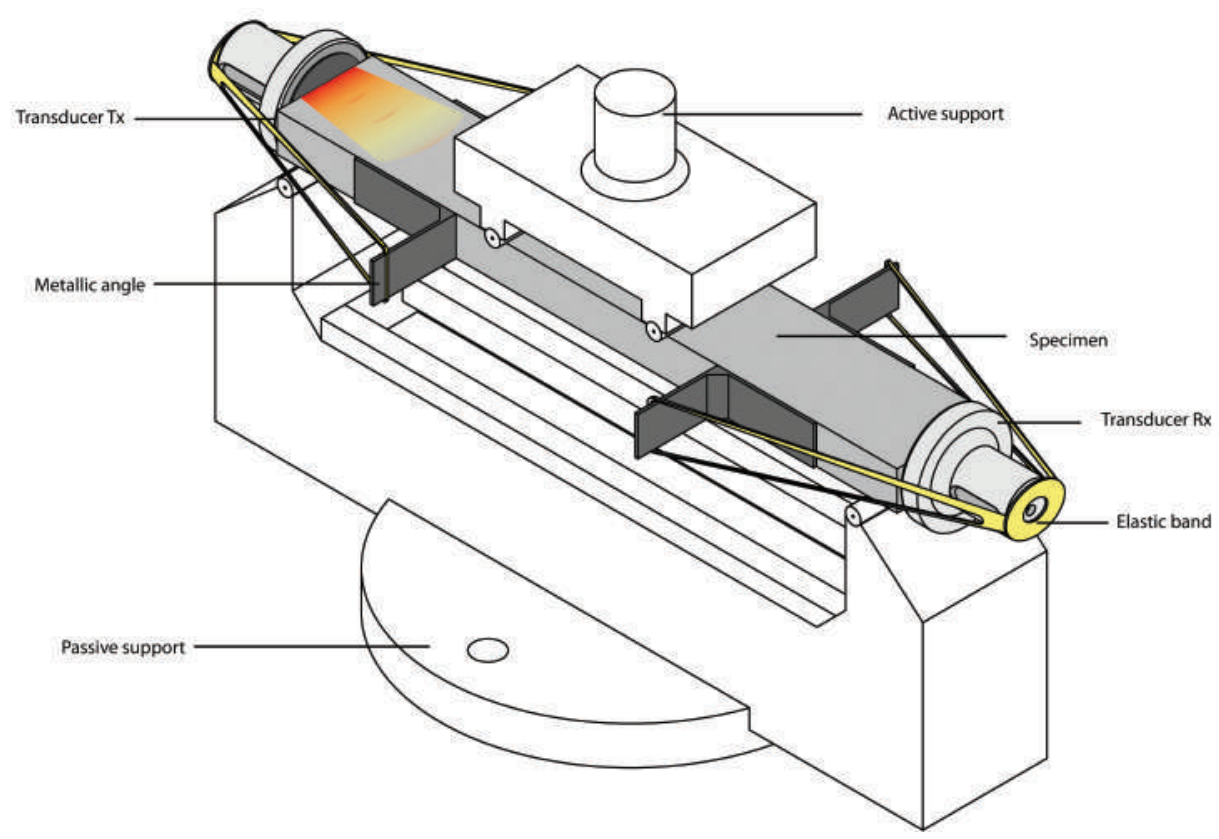

Figure 2: Experimental layout used in the ultrasonically monitored bending test. Four metallic angles are attached to the sides of the plate to support the elastic bands that keep constant the pressure between the faces of the transducers and the specimen.

The total received energy, $E_{\text {tot }}(t)$, is the energy of the received signal acquired by the oscilloscope, obtained as in Eq. (4).

$$
E_{\text {tot }}(t)[\mathrm{J}]=\int_{0}^{T} x_{t, r x}^{2}(\tau) d \tau
$$

The total attenuation of the material, $\alpha_{\text {tot }}(t)$, is obtained using Eq. (5). Given the analytical expression and the specific parameters of the transmitted signal (Eq. (1)), the transmitted energy $E_{t x}$ can be theoretically obtained using Eq. (6). In this paper, $E_{t x}$ equals $0.5 \mathrm{~mJ}^{1}$ and is constant throughout the test. Meanwhile, $E_{r x}(t)$ is obtained from Eq. (4). The global attenuation associated to the measurement equipment (transducers, amplifier, wires, acquisition module,...), $\alpha_{\text {equip }}$, is independent of the tested material and also constant throughout the test. In this paper, $\alpha_{\text {equip }}=-5 \mathrm{~dB}$. The value of the preamplifier must also be subtracted ( $\left.\alpha_{\text {preamp }}=40 \mathrm{~dB}\right)$.

$$
\begin{gathered}
\alpha_{\text {tot }}(t)[\mathrm{dB} / \mathrm{cm}]=\frac{E_{t x}-E_{r x}(t)-\alpha_{\text {equip }}-\alpha_{\text {preamp }}}{d_{\text {mat }}} \\
E_{t x}[J]=\int_{0}^{T_{w}} s_{t x}(\tau) d \tau=\frac{A_{t x}^{2} T_{w}}{2}+\frac{A_{t x}^{2}}{2} \int_{0}^{T_{w}} \cos \left(2 \pi\left(2 f_{0}\right) \tau+\pi\left(2 \Delta f_{\text {max }}\right) \tau^{2}\right) d \tau \approx \frac{A_{t x}^{2} T_{w}}{2}
\end{gathered}
$$

$$
\int_{0}^{1} \int_{w}^{T_{w}} \cos \left(2 \pi\left(2 f_{0}\right) \tau+\pi\left(2 \Delta f_{\max }\right) \tau^{2}\right) d \tau=\frac{\cos \left(\frac{2 \pi f_{0}^{2}}{\Delta f_{\max }}\right)\left(c\left(\frac{2\left(f_{0}+\Delta f_{\max } T_{w}\right)}{\sqrt{\Delta f_{\max }}}\right)-c\left(\frac{2 f_{0}}{\sqrt{\Delta f_{\max }}}\right)\right)+\sin \left(\frac{2 \pi f_{0}^{2}}{\Delta f_{\max }}\right)\left(s\left(\frac{2\left(f_{0}+\Delta f_{\max } T_{w}\right)}{\sqrt{\Delta f_{\max }}}\right)-s\left(\frac{2 f_{0}}{\sqrt{\Delta f_{\max }}}\right)\right)}{4 \sqrt{\Delta f_{\max }}} \text {, where } C(x)
$$
is the Fresnel $C$ integral and $S(x)$ is the Fresnel $S$ integral. 
The main advantage of the attenuation versus the total energy is that it is normalized by the input energy and the distance travelled through the material. That allows comparing the attenuation between different materials and experiments.

\subsubsection{Time and frequency dependent parameters}

The characteristic attenuation of the material can not only be analysed globally, $\alpha_{t o t}(t)$, but also in terms of the attenuation introduced in each frequency band. This two-dimensional variable depends on the analysis time and the frequency, $\alpha_{\text {mat }}(t, f)$. In this case, the attenuation can be modelled by Eq. (7) [16].

$$
\alpha_{\text {mat }}(t, f)=\frac{10 \log \left(S_{t x}(t, f)\right)-10 \log \left(S_{r x}(t, f)\right)-\alpha_{\text {equip }}^{\prime}(f)-\alpha_{\text {preamp }}}{d_{\text {mat }}}
$$

where $S_{t x}(t, f)$ is the energy spectral density (ESD) ${ }^{2}$ of the transmitted signal at time $t, S_{r x}(t, f)$ is that of the received signal, $d_{\text {mat }}$ is the distance of the specimen, and $\alpha_{\text {equip }}^{\prime}(f)$ is the attenuation due to the measurement equipment. Note that $\alpha_{\text {equip }}^{\prime}(f)$ is equivalent to $\alpha_{\text {equip }}$ in Eq. (5) when decomposed by frequency bands. This analysis considers the frequency response of the equipment used in the experiment. For further details about the calibration process and the computation of the ESD of a chirp signal, see [16].

The appearance of micro-cracks in the tested material leads to a non-linear effect known as Contact Acoustic Non-linearity (CAN) [21, 22]. This non-linear effect can be measured in terms of the variation of the amplitude of the higher harmonics. $\beta_{3}(t, f)$ is defined by Eq. 8 as the ratio between the amplitude of the third harmonic $\left(X_{r x}(t, 3 f)\right)$ and the fundamental harmonic $\left(X_{r x}(t, f)\right)$. The ratio between this parameter and the non-linear parameter before beginning the loading $(t=0)$ will be denoted by $\beta_{3}^{\%}(t, f)$, as in Eq. (9)

$$
\begin{aligned}
& \beta_{3}(t, f)=\frac{\left|X_{r x}(t, 3 f)\right|}{\left|X_{r x}(t, f)\right|^{3}} \\
& \beta_{3}^{\%}(t, f)=100 \frac{\beta_{3}(t, f)}{\beta_{3}(0, f)}
\end{aligned}
$$

In order to follow the same scheme as in previous papers [15], this parameter has also been analysed for a fixed working frequency, thus obtaining an index that depends only on the time. In the present paper, the chosen fixed frequency is $363 \mathrm{kHz}$ since the third harmonic appears in the center of the transducer band ( $\approx 1 \mathrm{MHz}$ ), being more sensitive to its location in the spectrum. This parameter, in the following denoted by $\beta_{3}(t, 363)$, complements the time dependent parameter analysis presented in the previous sub-section.

A common way to display results depending on these two independent variables is a time-frequency diagram. In these graphs, the $x$-axis corresponds to the time, the left $y$-axis indicates the stress/strain curve, the right $y$-axis is the frequency range, and the color axis represents the intensity of the analysed parameters, $\alpha_{m a t}(t, f)$ and $\beta_{3}(t, f)$.

\footnotetext{
${ }^{2}$ The energy spectral density, $S(f)$, of a finite time signal, $x(t)$, is defined as $S(f)=|X(f)|^{2}$, where $X(f)=\int_{\tau_{0}}^{\tau_{1}} x(\tau) e^{-i 2 \pi f \tau} d \tau$ is the Fourier Transform of $x(t)$.
} 


\section{Results and discussion}

In the first instance, due to the fact that the stress-strain curves are different and the characteristic events of the test occur at different time instants (depending on the specimen), no statistical values were extracted (mean, standard deviation, confidence intervals...) and a single plate was selected to follow the discussion and make it clear and understandable. The rest of the plots have been located in Appendix A, which shows the reproducibility and consistency of the results. Moreover, no corrections were made to the mechanical curves, in order to not disrupt the correlation between the mechanical and ultrasonic parameters.

In this section, the ultrasonic parameters and stress-strain curves were correlated. Firstly, the time dependent parameters, $v_{p}(t), E_{t o t}(t), \alpha_{t o t}(t)$, and $\beta_{3}^{\%}(t, 363)$, are represented and analysed (subsection 3.1 and Fig. 3). Then, the time and frequency dependent parameters, $\alpha_{m a t}(t, f)$ and $\beta_{3 f}^{\%}(t, f)$, are described in subsection 3.2.

\subsection{Time dependant parameters}

The results for the time dependent parameters are plotted in Fig. 3 using a double $y$-axis with the corresponding absolute values of each parameter.

Fig. 3a shows the evolution of the velocity of the chirp signal during the test. The velocity parameter, $v_{p}(t)$, stays constant in the initial stage of the test (supports and specimen's surface coupling stabilization) and for most of

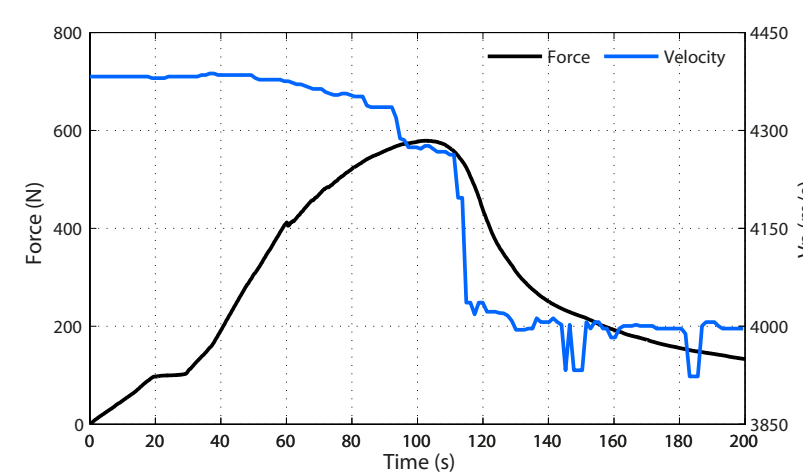

(a)



(c)

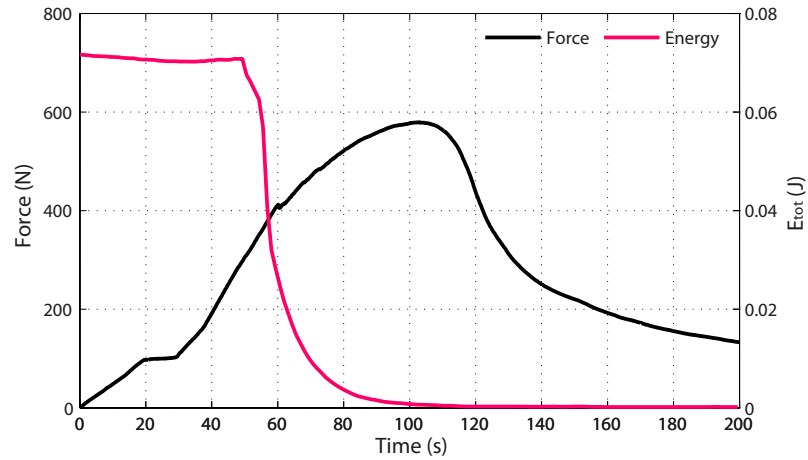

(b)

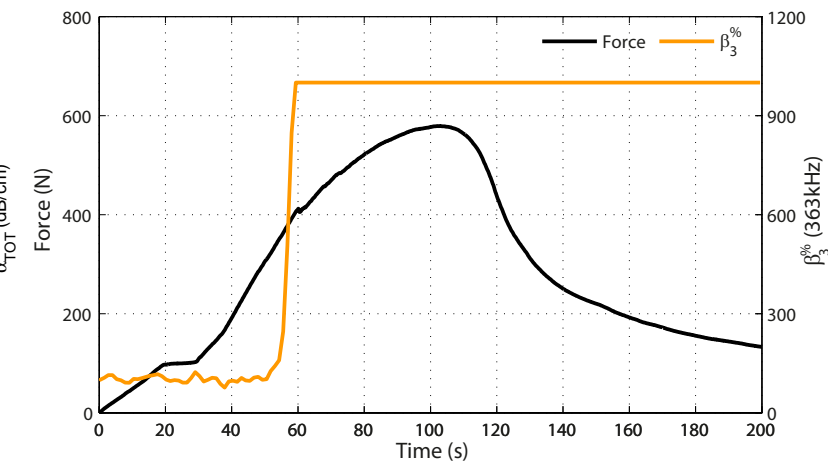

(d)

Figure 3: Comparison between absolute values of time dependent ultrasonic parameters and stress-strain curve of one specimen: (a) velocity of the chirp signal, (b) energy of the received signal, (c) total attenuation and (d) non-linear parameter $\beta_{3}^{\%}$ measured at $363 \mathrm{KHz}$. 
the elastic step of the stress-strain curve (0-50 s). When the first micro-cracks begin to appear (50-60 s), $v_{p}(t)$ decays slightly $(\approx 10 \mathrm{~m} / \mathrm{s}$ ), and after that, goes down to values around $4000 \mathrm{~m} / \mathrm{s}$ and becomes unstable due to the low signal-to-noise ratio (SNR) (failure when estimating $\tau_{t, a}$ ).

Figure 3b shows the trend of energy of the received wave. Three zones can be clearly identified in the evolution of $E_{\text {tot }}(t)$. In the first zone (0-50 s), the parameter stays constant while the specimen deforms. In the second zone (55-100 s), the $E(t)$ curve starts to decay to values close to zero before the cracking process starts (60 s) due to the micro-cracking process previous to the first large crack. After that (100-end of the test), $E(t)$ stays at zero values because the section of the plate is getting progressively smaller.

The attenuation of the material, $\alpha_{t o t}(t)$, is plotted in Fig. 3c. There is a trend opposite to that obtained for the previous parameters. In the first zone, $\alpha_{t o t}(t)$ stays constant (close to $2 \mathrm{~dB} / \mathrm{cm}$ ), however, before the appearance of the first crack (55 s), it rises to high values $(2.7 \mathrm{~dB} / \mathrm{cm})$ and stays constant until the end of the test.

As mentioned in Section 2, $\beta_{3}^{\%}(t, 363)$ was monitored because it is very sensitive to crack formation. In order to calculate $\beta_{3}^{\%}(t)$ it is necessary to fix a discrete frequency since the emitted signal contains information on several frequencies. The specific frequency $363 \mathrm{kHz}$ was used to analyse the generation of high-frequency harmonics because the third harmonic appears in the center of the transducer's band ( $\approx 1 \mathrm{MHz}$ ), being more sensitive to its location in the spectrum. Figure $3 \mathrm{~d}$ shows the evolution of $\beta_{3}^{\%}(t)$ as the test progresses. It can be noticed that this parameter dramatically increases (a variation of 1000\%) in the critical zone, after the beginning of the cracking process of the plate $(\approx 55 \mathrm{~s})$. The fact that $\beta_{3}^{\%}(t)$ changes quickly in the beginning of the cracking process indicates premature damage in the specimen (in the elastic step of the stress-strain curve) warning of the damage that the specimen has already suffered.

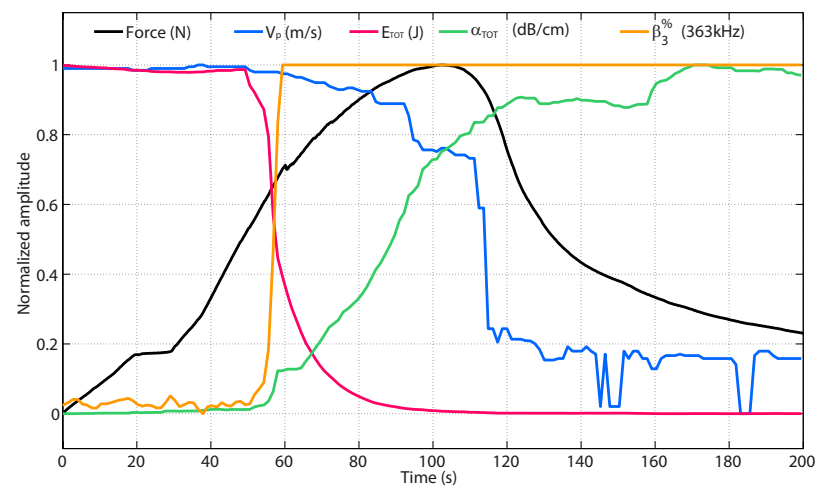

Figure 4: Comparison of the normalized values of the time dependent ultrasonic parameters and the stress-strain curve of one GRC specimen.

In order to compare the time dependent parameters, their normalized values were calculated (Fig. 4). As can be observed, $v_{p}(t)$ does not show relevant information in the stage previous to the first cracks, showing a slow detection of the damage suffered by the plate. However, $E_{\text {tot }}(t)$ and $\alpha_{t o t}(t)$ are more sensitive, showing significant variations previous to the plastic step of the stress-strain curve. Significantly, $\beta_{3}^{\%}(t, 363)$ exhibits an interesting behaviour, marking perfectly the instant when the specimen begins to suffer initial damage. This means that the 
non-linear parameter is the most sensitive way to identify the change from the elastic to plastic zone in GRC.

\subsection{Time and frequency dependent parameters}

The results for the parameters depending on both the time and the frequency are shown in Figs 5 and 6 . The $x$-axis indicates the test time, the left $y$-axis the force (stress-strain curve) and the right $y$-axis the analysed frequency range. The parameter under study is shown with different colours and its respective gradients.

Figure 5a shows the evolution of $\alpha_{\text {mat }}(t, f)$ as the bending test progresses. In the first instance and for a fixed value of $t$, the attenuation parameter rises with the increase of the analysed frequency, as suggested in most of the literature and in previous papers $[6,15,20]$. For each frequency, this means, for a fixed frequency $f$, the parameter of attenuation also rises along the experiment. A similar trend can be observed in Fig. 3c for $\alpha_{t o t}(t)$, which only depends on the test time. By means of analysing $\alpha_{\text {mat }}(t, f)$, there can be obtained the complete material response to different injected frequencies along the experiment time. The attenuation remains constant during the stabilization stage $(0-30 \mathrm{~s})$ and for most of the elastic step (30-60 s). Near the first crack, at the end of the elastic step (50-55 s), the attenuation patterns change and it starts to rise to higher values due the initial phase of the micro-cracking process. Once the plate is in the plastic stage (65-90 s), the attenuation for frequencies under $600 \mathrm{kHz}$ rises. Slightly before the maximum stress point $(\approx 90 \mathrm{~s})$ the attenuation for higher frequencies (over $600 \mathrm{kHz}$ ) also increases, to reach its maximum attenuation value. From the maximum stress point $(\approx 110 \mathrm{~s})$ on, the attenuation patterns keep constant until the end of the test, where the effective cross section of the plate is low. The time-frequency diagrams for the rest of the tested plates are shown in Appendix A.

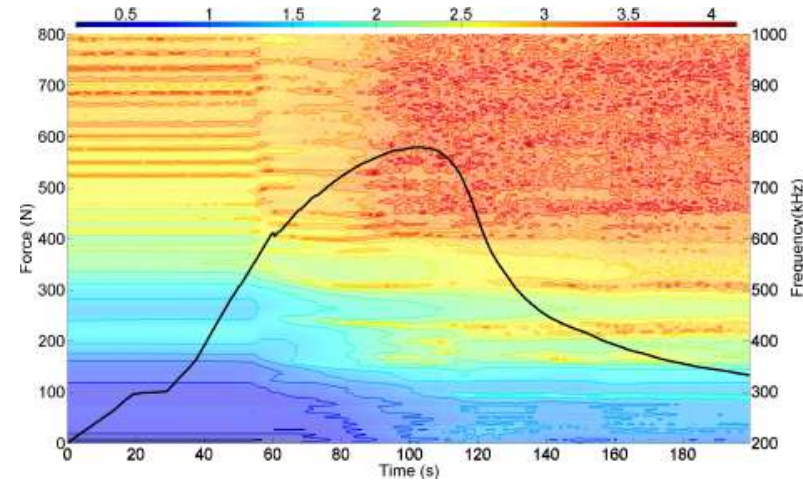

(a)



(b)

Figure 5: Time-frequency diagrams of the parameters related to the attenuation. The $x$-axis indicates the test time, the left $y$-axis the force (stress-strain curve) and the right $y$-axis the analysed frequency range. The parameter under study is shown with different colours and its respective gradients. a) Time-frequency diagram of the $\alpha_{\text {mat }}(t, f)[\mathrm{dB} / \mathrm{cm}]$. b) Time-frequency diagram of the normalized $\Delta_{t} \alpha_{\text {mat }}(t, f)$.

The interest of this study lies in finding and highlighting the changes in the ultrasonic parameters with the progress of the bending test and in correlating these changes with the events produced in the specimen. As has been observed, $\alpha_{\text {mat }}(t, f)$ seems to be sensitive to any change in the mechanical properties of the material under study. Nonetheless, the representation of the absolute values of $\alpha_{\text {mat }}(t, f)$ may hide some notable changes. Thus, a further analysis of the attenuation, considering the sensitivity of each frequency, is proposed. Figure 5b plots 
the variation of the attenuation with respect to time, normalized over the maximum value for each frequency, $\Delta_{t} \alpha_{\text {mat }}(t, f)$. This graph allows identifying the main events happening during the bending test: the first crack $(\approx 55 \mathrm{~s})$ and the maximum stress point of the specimen $(\approx 110)$, even by a certain time in advance of the mechanical curve. Note that this normalized representation allows comparing the attenuation levels regardless of the working frequency. Changes in $\Delta_{t} \alpha_{\text {mat }}(t, f)$ for the first crack are observed for all studied frequencies, although frequencies under $600 \mathrm{kHz}$ are more sensitive. Additionally, for the maximum stress point, the most sensitive frequencies frequencies are lower than $400 \mathrm{kHz}$. In Appendix A, time-frequency diagrams for the rest of the tested plates are shown. As can be observed, those attenuation results present similar patterns by overcoming the differences between specimens and experiences.

Figure 6 shows the corresponding graph for $\beta_{3}^{\%}(t, f)$. As could be observed in the previous figures (Fig. 3d), $\beta_{3}^{\%}(t, 363)$ was very sensitive to the crack formation, varying its value drastically when the specimen is damaged by the formation of the first crack. In this figure, this behaviour can be seen for all frequencies contained in the frequency range chosen according to the location of the third harmonic and the transducer response as mentioned before (300-500 kHz). This non-linear parameter remains constant during the stabilization stage for all frequencies, as do the rest of the ultrasonic parameters.

When the stress-strain curve approaches the cracking zone, $\beta_{3}^{\%}(t, f)$ increases rapidly for several frequencies, reaching higher values before the cracks promoted by the deformation of the specimen appear. Since each stressstrain curve is unique, each time-frequency diagram varies (see Appendix A), depending on the specimen but always following the same pattern (a rapid increase before the beginning of the plastic step).

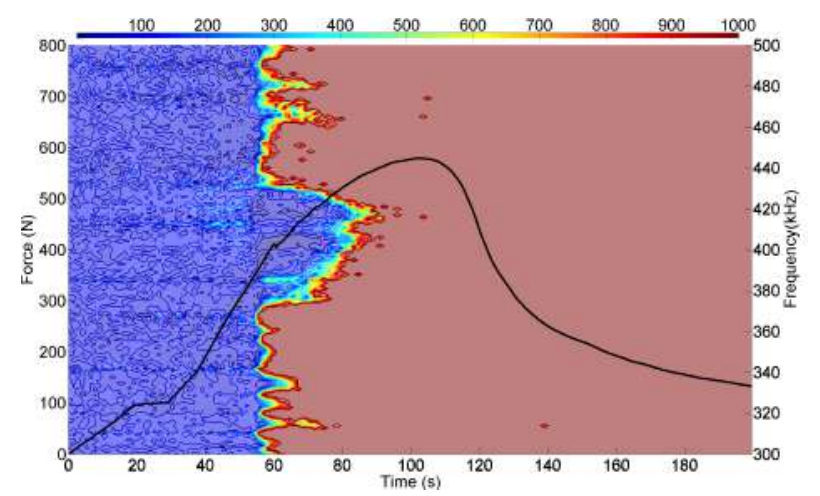

Figure 6: Time-frequency diagram of the non-linear parameter $\beta_{3}^{\%}(t, f)$. The $x$-axis indicates the test time, the left $y$-axis the force (stressstrain curve) and the right $y$-axis the analysed frequency range. The parameter under study is shown with different colours and its respective gradients.

Regarding the obtained results, it can be concluded that the attenuation time-frequency diagram gives information about the continuous changes between the different steps of the test. However, the non-linear parameter seems to be the best for an analysis that will robustly characterize the cracking instant.

The sensitivity to inhomogeneities (pores, cracks...) of each frequency depends not only on the dimension of the discontinuity but also the direction, disposition, separation between faces, etc. For this reason, it is very important 
to have the proposed $\alpha_{\text {mat }}(t, f)$ and $\beta_{3 f}^{\%}(t, f)$ time-frequency diagrams to accurately detect when the element under test is being critically damaged.

\section{Conclusions}

In this paper, a complete ultrasonic monitoring of GRC plates under bending tests was addressed. A recently proposed procedure based on broadband signals (chirp) was used in order to measure different ultrasonic parameters in a wide range of frequencies. This new method of monitoring bending tests provides a wealth of information about the material behaviour in terms of the attenuation, energy, velocity, and generation of non-linear harmonics in the signal. In order to support the previous results and provide new information, the analysis has been divided into two parts: those parameters dependant only on time, and those dependent on both time and frequency. In the first group, the evolution of the velocity roughly characterizes the main two events happening during the test: the first large crack and the maximum stress point. However, the energy and attenuation of the chirp signal were more sensitive, showing a significant variation previous to the plastic step of the stress-strain curve, attenuation being more appropriate for comparing different materials and tests. It was conspicuous that the non-linearity in the generation of high harmonics exhibited an interesting behaviour, marking perfectly the instant when the specimen was suffering damage. In the second part, the attenuation and non-linearity were analysed in terms of time and frequency, giving more information about the material properties duing the progress of the test. The study of this variation of the attenuation along time for each fixed frequency has been proposed. This new normalized approach has allowed comparing the trends between different working frequencies, as well as significantly detecting both the first large crack and the maximum stress point. The non-linearity had a sudden change as the micro-cracking began, pointing perfectly to the time instant when the specimen is being critically damaged.

This paper has demonstrated the suitability of ultrasonic broadband signals to characterize fiber-reinforced cementitious composites under bending stress since it allows simultaneously analysing some of the traditional ultrasonic parameters in the frequency domain. This approach might open up a new line of research in any further application where the acquisition time is a critical variable.

\section{Acknowledgements}

The authors want to acknowledge the financial support of the Ministerio de Economía y Competitividad (MINECO), Spain, and FEDER funding (Ondatest Projects: BIA2014-55311-C2-1-P and BIA2014-55311-C2-2-P). 


\section{Appendix A. Figures}

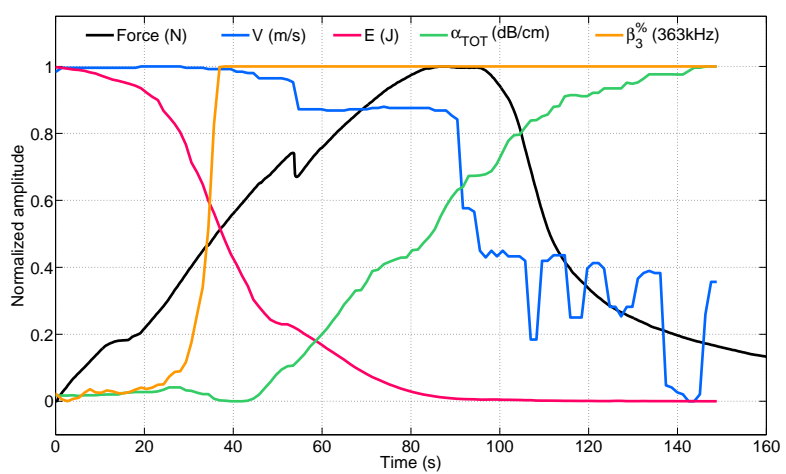

(a)

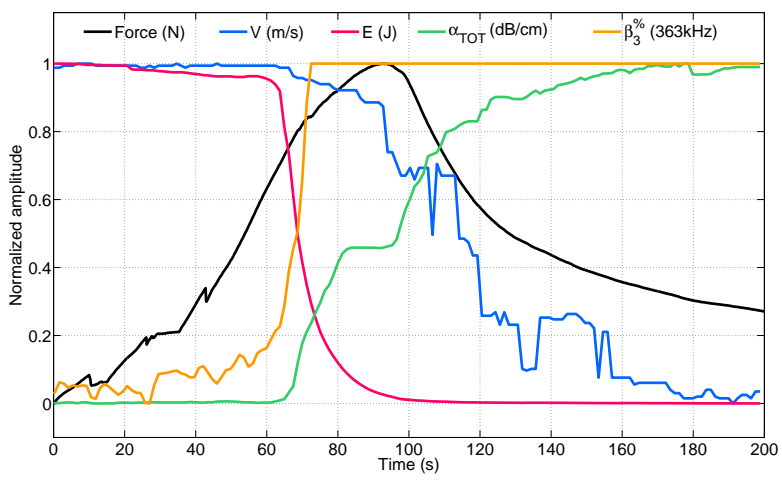

(c)

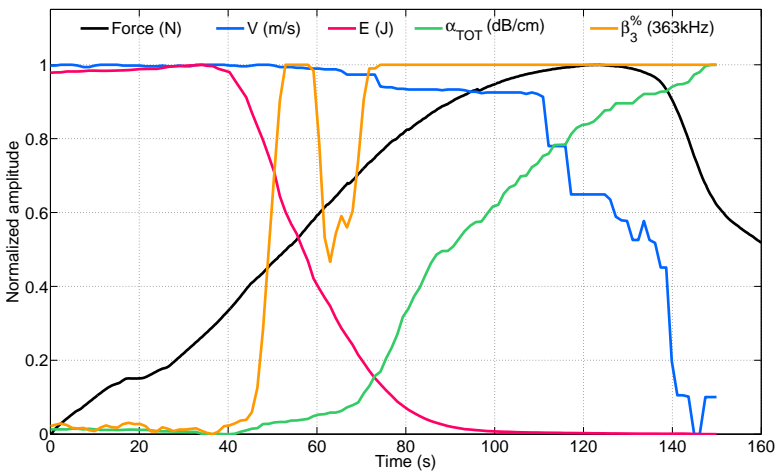

(b)

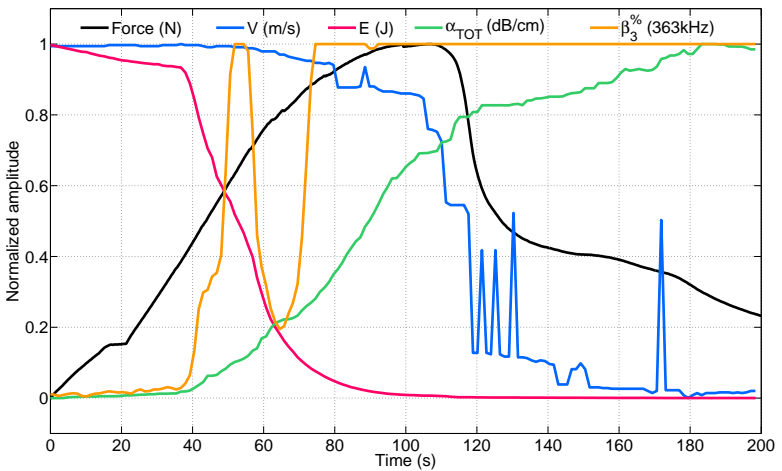

(d)

Figure A.1: Normalized time-dependent parameters for the rest of the tested specimens. 


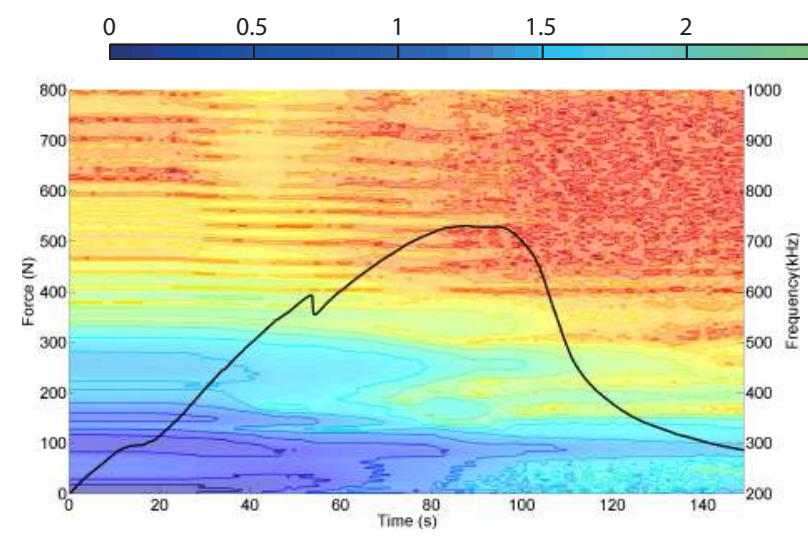

(a)

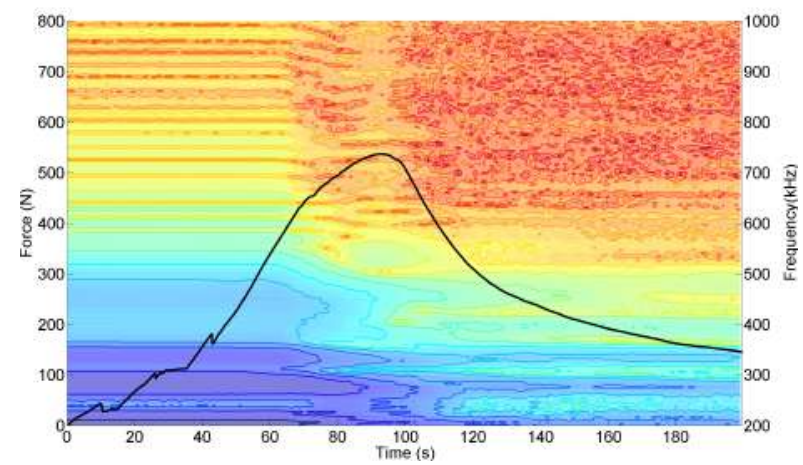

(c)
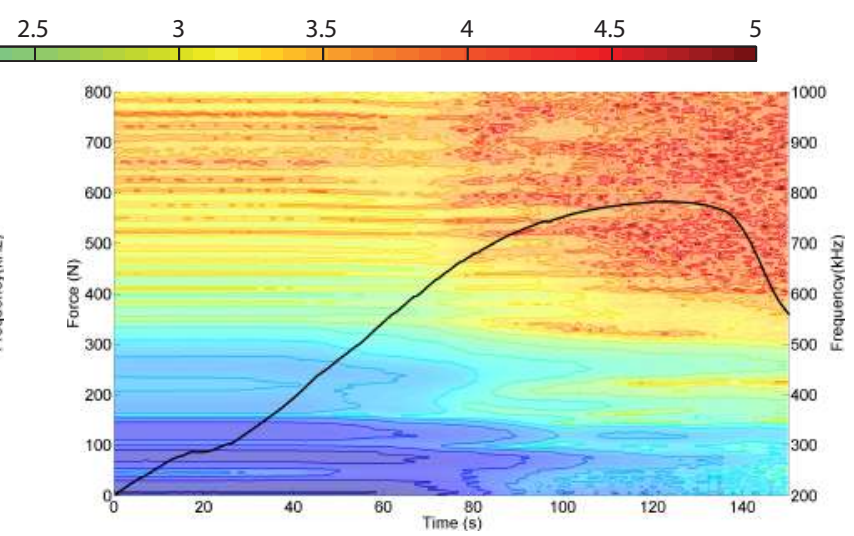

(b)

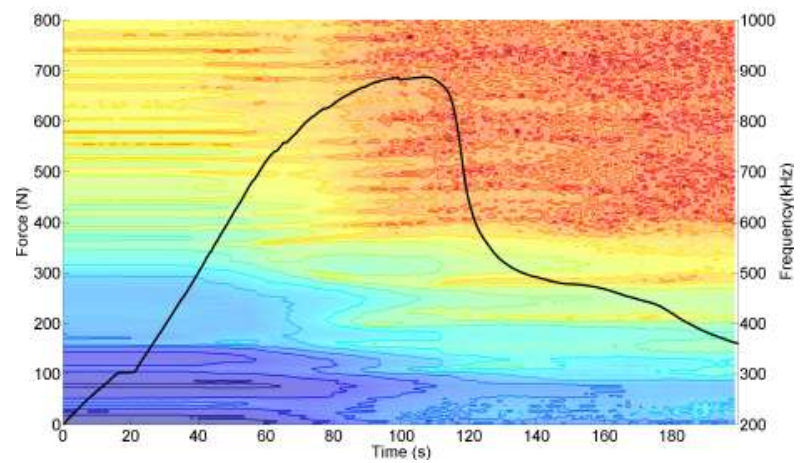

(d)

Figure A.2: Time-frequency diagrams of the attenuation, $\alpha_{m a t}(t, f)$. The $x$-axis indicates the time, the left $y$-axis the force (stress-strain curve), and the right $y$-axis the analysed frequency range. The parameter under study is shown with different colours and its respective gradients for the rest of the analyzed probes. 


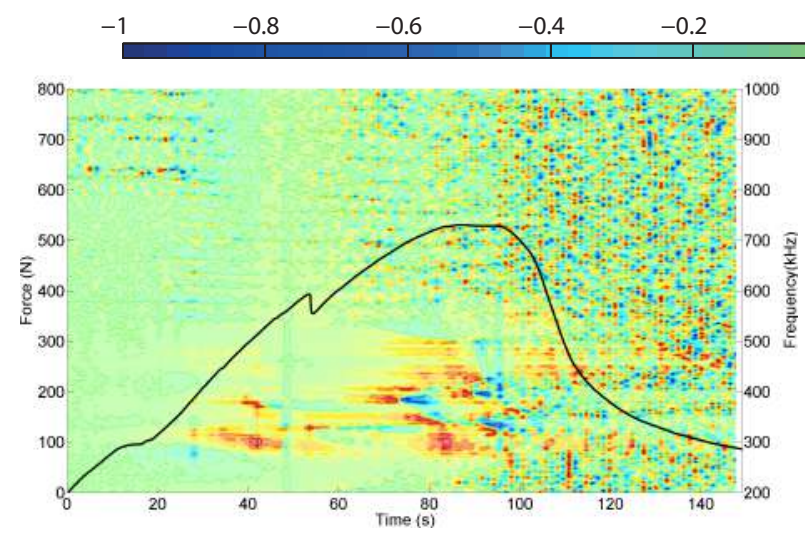

(a)

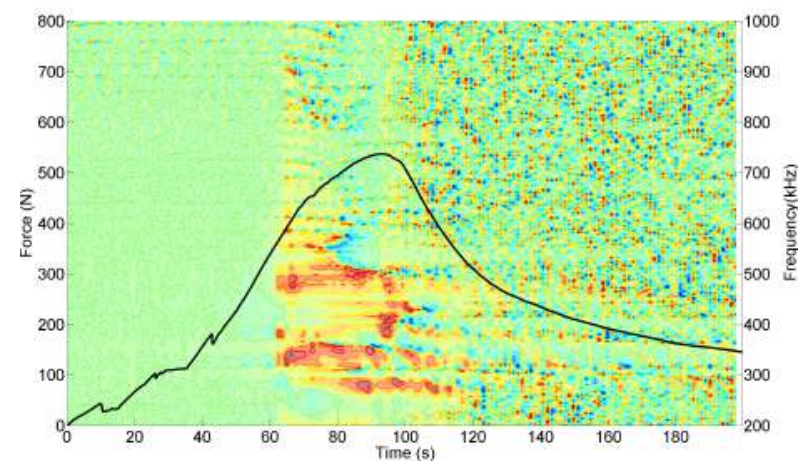

(c)

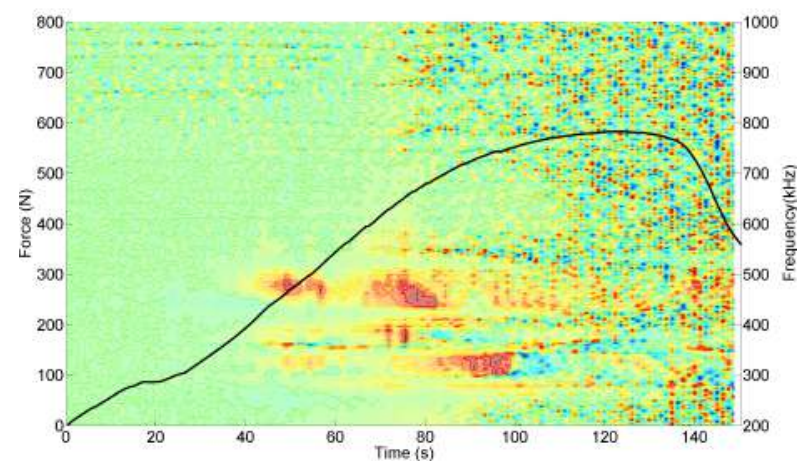

(b)

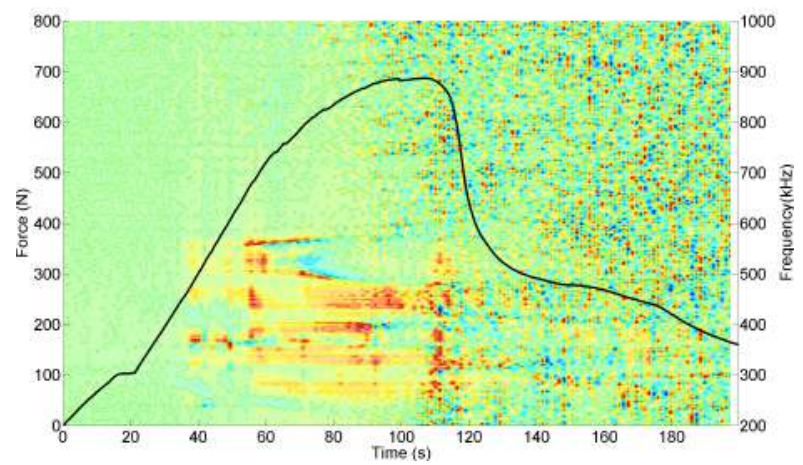

(d)

Figure A.3: Time-frequency diagrams of variation of the attenuation, $\Delta_{t} \alpha_{m a t}(t, f)$. The $x$-axis indicates the time, the left $y$-axis the force (stress-strain curve), and the right $y$-axis the analysed frequency range. The parameter under study is shown with different colours and its respective gradients for the rest of the analyzed probes. 


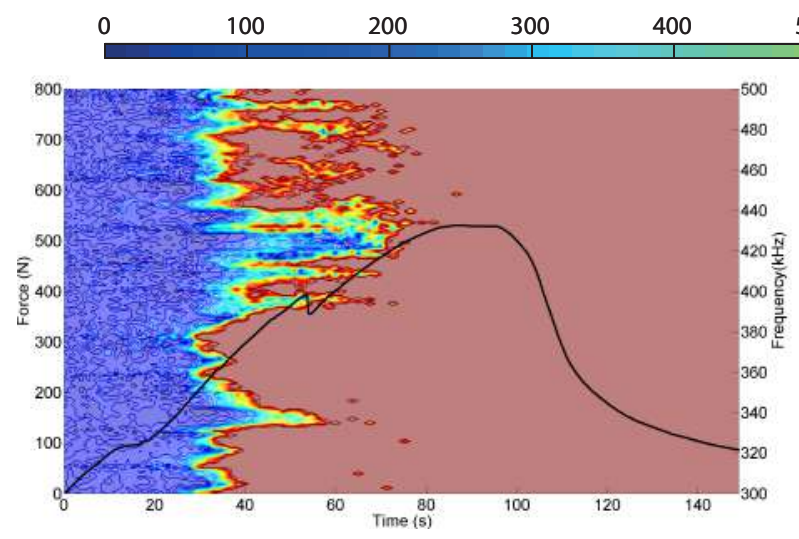

(a)

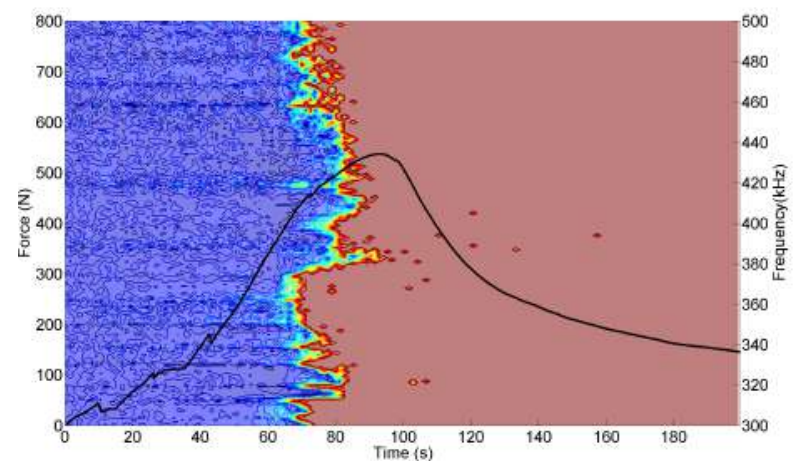

(c)

$\begin{array}{lllll}500 & 600 & 700 & 800 & 900\end{array}$

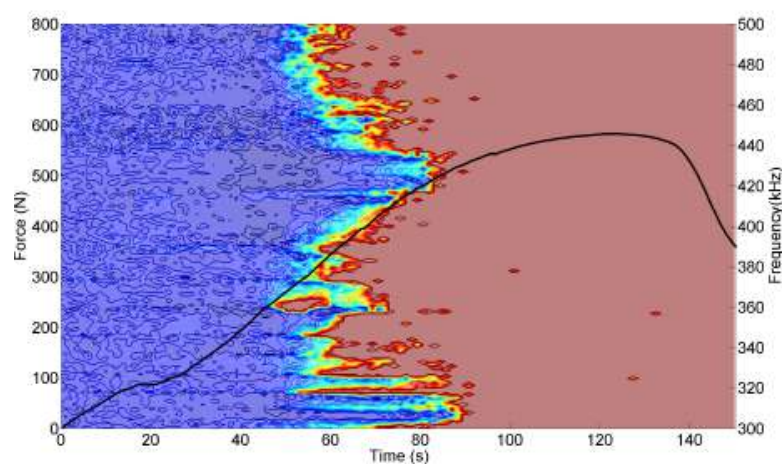

(b)

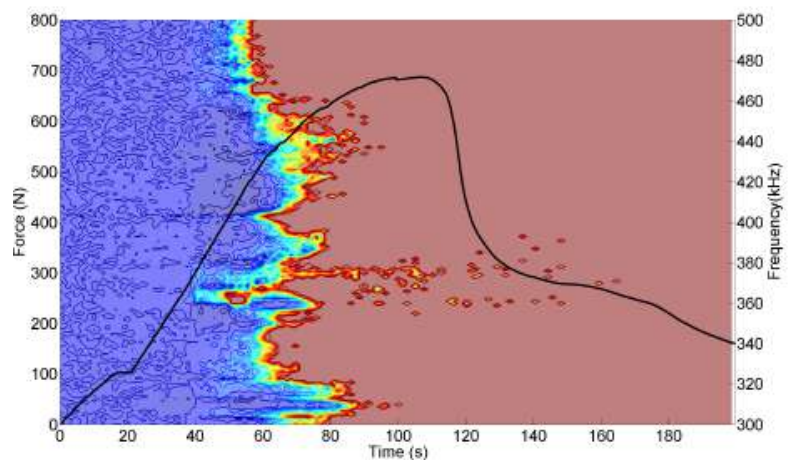

(d)

Figure A.4: Time-frequency diagrams of non-linear parameter, $\beta_{3}^{\%}(t, f)$. The $x$-axis indicates the time, the left $y$-axis the force (stress-strain curve), and the right $y$-axis the analysed frequency range. The parameter under study is shown with different colours and its respective gradients for the rest of the analyzed probes. 


\section{References}

[1] A. J. Majumdar, J. Ryder, Glass fibre reinforcement of cement products, Garston, England: Building Research Station, 1968.

[2] A. Bentur, Fibre Reinforced Cementitious Composites, Taylor \& Francis, 1990.

[3] V. Malhotra, N. Carino, Handbook on Nondestructive Testing of Concrete Second Edition, CRC Press, 2003.

[4] J. A. Bogas, M. G. Gomes, A. Gomes, Compressive strength evaluation of structural lightweight concrete by non-destructive ultrasonic pulse velocity method, Ultrasonics 53 (5) (2013) 962-972.

[5] A. Jain, A. Kathuria, A. Kumar, Y. Verma, K. Murari, Combined Use of Non-Destructive Tests for Assessment of Strength of Concrete in Structure, Procedia Engineering 54 (2013) 241-251.

[6] T. P. Philippidis, D. G. Aggelis, Experimental study of wave dispersion and attenuation in concrete, Ultrasonics 43 (7) (2005) $584-95$.

[7] S. Popovics, J. L. Rose, J. S. Popovics, The behaviour of ultrasonic pulses in concrete, Cement and Concrete Research 20 (2) (1990) 259-270.

[8] L. Vergara, J. Gosálbez, J. Fuente, R. Miralles, I. Bosch, Measurement of cement porosity by centroid frequency profiles of ultrasonic grain noise, Signal Processing 84 (12) (2004) 2315-2324.

[9] L. Vergara, R. Miralles, J. Gosálbez, F. Juanes, L. Ullate, J. Anaya, M. Hernández, M. Izquierdo, NDE ultrasonic methods to characterise the porosity of mortar, NDT \& E International 34 (8) (2001) 557-562.

[10] D. Aggelis, T. Philippidis, Ultrasonic wave dispersion and attenuation in fresh mortar, NDT \& E International 37 (8) (2004) $617-631$.

[11] F. Moradi-Marani, P. Rivard, C. Lamarche, S. Kodjo, Evaluating the damage in reinforced concrete slabs under bending test with the energy of ultrasonic waves, Construction and Building Materials 73 (2014) 663-673.

[12] H. Ogi, T. Hamaguchi, M. Hirao, In-situ monitoring of ultrasonic attenuation during rotating bending fatigue of carbon steel with electromagnetic acoustic resonance, Journal of Alloys and Compounds 310 (1-2) (2000) 436-439.

[13] S. Sharma, A. Mukherjee, Monitoring freshly poured concrete using ultrasonic waves guided through reinforcing bars, Cement and Concrete Composites 55 (2015) 337 - 347, ISSN 0958-9465.

[14] H. von Daake, D. Stephan, Setting of cement with controlled superplasticizer addition monitored by ultrasonic measurements and calorimetry, Cement and Concrete Composites 66 (2016) $24-37$.

[15] V. Genovés, J. Gosálbez, R. Miralles, L. Soriano, J. Payá, Ultrasonic monitoring on glass fiber reinforced cement (GRC) bending test, Materials Characterization 90 (2015) 149-158.

[16] V. Genovés, J. Gosálbez, A. Carrión, R. Miralles, J. Payá, Optimized ultrasonic attenuation measures for non-homogeneous materials, Ultrasonics 65 (2016) 345 - 352, ISSN 0041-624X.

[17] British Standard, Precast concrete products-Test method for glass-fibre reinforced cement—Part 5. Measuring bending strength, 'Complete bending test' method .

[18] J. Krautkrämer, H. Krautkrämer, Ultrasonic Testing of Materials, Springer, 1983.

[19] P. Gaydecki, F. Burdekin, The propagation and attenuation of medium-frequency ultrasonic waves in concrete: A signal analytical approach, Measurement Science 126 (1992) 400.

[20] M. Molero, I. Segura, S. Aparicio, M. Hernández, M. Izquierdo, On the measurement of frequency-dependent ultrasonic attenuation in strongly heterogeneous materials, Ultrasonics 50 (8) (2010) 824-8.

[21] A. Shah, Y. Ribakov, Non-linear ultrasonic evaluation of damaged concrete based on higher order harmonic generation, Materials \& Design 30 (10) (2009) 4095-4102.

[22] A. Shah, Y. Ribakov, Non-destructive evaluation of concrete in damaged and undamaged states, Materials \& Design 30 (9) (2009) 3504-3511. 